\title{
Individualized Treatment Strategy with Small-Molecular Inhibitors in Acute Myeloid Leukemia with Concurrent FLT3-ITD and FLT3-TKD Mutation
}

Harald Polzer ${ }^{1-4 *}$, Hanna Janke ${ }^{1,2}$, Stephanie Schneider ${ }^{1}$, Wolfgang Hiddemann ${ }^{1-4}$, Marion Subklewe ${ }^{1}$ and Karsten Spiekermann ${ }^{1-4}$

${ }^{1}$ Department of Internal Medicine III, University Hospital Großhadern, Ludwig-Maximilians-University Munich, Germany

${ }^{2}$ Clinical Cooperative Group "Leukemia", Helmholtz Center Munich, Germany

${ }^{3}$ German Cancer Consortium (DKTK), Heidelberg, Germany

${ }^{4}$ German Cancer Research Center (DKFZ), Heidelberg, Germany

\begin{abstract}
FLT3 is a frequently mutated gene in acute myeloid leukemia that encodes a receptor tyrosine kinase. We report the case of a patient with FLT3-ITD positive secondary acute myeloid leukemia after treatment for breast cancer. Due to poor response to induction therapy and relapse before consolidation therapy we started a palliative treatment with the tyrosine kinase inhibitor sorafenib. After initial response clinical resistance occurred. Sequencing showed an additional FLT3-TKD mutation. Sunitinib effectively inhibited FLT3-ITD/TKD mutated cells in vitro and induced a reduction of blasts and prolonged survival in vivo. Individualized tyrosine kinase inhibitor therapy may prolong survival in selected patients with FLT3-ITD+ acute myeloid leukemia.
\end{abstract}

Keywords: Acute myeloid leukemia; FLT3; Internal tandem duplication; Tyrosine kinase domain; Tyrosine kinase inhibitor (TKI)

\section{Introduction}

"FMS-like tyrosine kinase 3" (FLT3) belongs to the receptor tyrosine kinase (RTK) class III receptors and plays a critical role in proliferation of early hematopoietic stem and progenitor cells. FLT3 mutations are frequently found in AML and a deregulation of the FLT3 receptor plays a major role in the pathogenesis of leukemia. Most common mutations are internal tandem duplications (FLT3ITD) and mutations in the second tyrosine kinase (FLT3-TKD) [1]. In contrast to the strong phenotype induced by FLT3-ITD in vitro and their prognostic impact on clinical course, FLT3-TKD mutations show a weaker transforming potential [2]. However, acquired FLT3-TKD mutations can cause resistance against tyrosine kinase inhibitors (TKI) in FLT3-ITD positive AML [3-5].

\section{Materials and Methods}

\section{Cell culture}

The cell line $\mathrm{Ba} / \mathrm{F} 3$ was obtained from the Deutsche Sammlung von Mikroorganismen und Zellkulturen GmbH (DSMZ; Braunschweig, Germany), and maintained in RPMI 1640 with $10 \%$ fetal bovine serum and 10\% WEHI conditioned medium as source of Interleukin 3 (IL3).

\section{Growth factors, antibodies and inhibitors}

The recombinant human FLT3-Ligand (FL) and murine IL3 were purchased from Immunotools (Friesoythe, Germany). Sorafenib, sunitinib and AC220 were obtained from Selleck Chemicals (Munich, Germany).

\section{DNA constructs and in vitro mutagenesis}

The human FLT3-WT and FLT3-ITD-W51 constructs were kindly provided by Gary Gilliland (Boston Harvard Medical School, MA, USA). FLT3-ITD-W51: 7 amino acids (REYEYDL) inserted between amino acids 601 and 602 of human FLT3-WT. FLT3 point mutation D835Y was introduced as described previously [6]. Transient transfection of Phoenix Eco cells and stable transduction of $\mathrm{Ba} / \mathrm{F} 3$ cells. These experiments were performed as described previously [6].

\section{Proliferation assay}

For the proliferation assay $4 \times 104 / \mathrm{ml} \mathrm{Ba} / \mathrm{F} 3$ cells were seeded in growth medium in presence or absence of inhibitors, FL or IL-3. Viable cells were counted after $72 \mathrm{~h}$ by trypan blue exclusion using the cell viability analyzer Vi-Cell AS (Beckman Coulter). Experiments were replicated independently four times.

\section{Clinical Case}

We present the case of a female patient with metastasized breast cancer diagnosed in 2010. In 2011 local progress in the bone and new hepatic metastases were diagnosed and therapy with docetaxel was initiated. In a routine screen in August 2012 blasts were seen in the blood count and the patient was administered to our clinic. The 54 years old patient reported of reduced performance status and night sweats, but no fevers, weight loss or signs of infection. The peripheral blood count showed a leukocytosis of $21.7 \mathrm{G} / \mathrm{l}$ with $60 \%$ myeloid blasts. The bone marrow blast count was about $90 \%$ and genetics revealed a normal karyotype and the presence of a FLT3-ITD. Due to the previous chemotherapy a therapy-related AML according to WHO was diagnosed. An induction therapy with cytarabine and daunorubicin $(7+3)$ was performed in September 2012 [7]. At day 18 of the induction therapy blast cells persisted in the bone marrow to $49 \%$. A second induction therapy with high-dose cytarabine and mitoxantrone (HAM) was started in October 2012 [8]. On day 10 after HAM no blasts were seen in the aplastic bone marrow. However, in December 2012 the patient relapsed with $32 \%$ blasts in the bone marrow. Because

*Corresponding author: Harald Polzer, Department of Internal Medicine III, University Hospital Großhadern, Ludwig-Maximilians-University Munich, Germany, Tel: 49-89-440043989; Fax: 49-89-4400-77565; E-mail: harald.polzer@med.uni-muenchen.de

Received September 08, 2015; Accepted October 06, 2015; Published October 20, 2015

Citation: Polzer H, Janke H, Schneider S, Hiddemann W, Subklewe M, et al (2015) Individualized Treatment Strategy with Small-Molecular Inhibitors in Acute Myeloid Leukemia with Concurrent FLT3-ITD and FLT3-TKD Mutation. J Clin Case Rep 5: 622. doi:10.4172/2165-7920.1000622

Copyright: $\odot 2015$ Polzer $\mathrm{H}$, et al. This is an open-access article distributed under the terms of the Creative Commons Attribution License, which permits unrestricted use, distribution, and reproduction in any medium, provided the original author and source are credited. 
of the poor response to induction therapy, the presence of a FLT3-ITD and the concurrent metastasized breast cancer an off-label therapy with sorafenib was started [5]. Initial dosage was $800 \mathrm{mg}$ daily which was reduced due to haematotoxicity in the course of the treatment. Blast count in the hypocellular bone marrow was at 75\% in February 2013, most probably due to a pause of the treatment during January because of therapy-related pancytopenia. In the following sorafenib $200 \mathrm{mg}$ was taken every second day from March to June 2013. Blast count in the hypocellular bone marrow was at $12 \%$ in April 2013 with persisting FLT3-ITD allele burden. Dose was escalated to $200 \mathrm{mg}$ daily in June because blast count in the peripheral blood was increasing. In July 2013 a progress of the AML under sorafenib treatment with rise of the $\mathrm{LDH}$, leukocytosis, thrombocytopenia and $29 \%$ blasts in the peripheral blood was diagnosed. Molecular genetic analysis showed a subclone with trisomy 8 and 13 as well as an additional FLT3 point mutation at amino acid D835. The treatment with sorafenib was stopped and the patient received cytarabine for 3 days.

To identify a non-cross resistant FLT3 TKI we performed an in vitro screen in FLT3-ITD and FLT3-TKD mutated receptor positive IL-3 dependent Ba/F3 cells. We analyzed the inhibition of cell proliferation of three selective small molecule kinase inhibitors of FLT3 in vitro. In addition to sorafenib we used sunitinib as another approved TKI and AC220 which is currently the most promising compound in clinical AML trials. We evaluated concentrations up to $250 \mathrm{nmol} / \mathrm{l}$ for AC220, up to $1000 \mathrm{nmol} / \mathrm{l}$ for sorafenib and up to $250 \mathrm{nmol} / \mathrm{l}$ for sunitinib (Figure 1). In presence of IL-3 the proliferation of the $\mathrm{Ba} /$ F3 cells was not affected (data not shown) but all three compounds potently inhibited the cell proliferation driven by FLT3-ITD in absence of IL-3 at a very low nanomolar range (IC50 AC220: $<0.5 \mathrm{nM}$; IC50 sorafenib: $2.1 \mathrm{nM}$; IC50 sunitinib: $2.8 \mathrm{nM}$; (Figure 2). In the case of the
FLT3-TKD D835Y mutation sorafenib had a half maximal inhibitory concentration (IC50) of $49 \mathrm{nM}$ in Ba/F3 cells. This was more than 10 fold higher compared to AC220 (IC50: $2.3 \mathrm{nM}$ ) and sunitinib (IC50: $3 \mathrm{nM}$ ) (Figure 2D). The cell proliferation of the FLT3 double mutant FLT3-ITD-D835Y was potently inhibited by AC220 (IC50: $38 \mathrm{nM}$ ) and sunitinib (IC50: $22 \mathrm{nM}$ ) but showed strong resistance against sorafenib treatment (IC50: $795 \mathrm{nM}$ ).

According to our in vitro analysis we started a treatment with sunitinib in August 2013. $50 \mathrm{mg}$ per day is the recommended dose in treatment of renal cell cancer and gastrointestinal stromal tumors. At this dose a phase I study has shown plasma levels of 50-100 ng/ $\mathrm{ml}$ in treatment of AML patients [9]. A reduction of blasts in the peripheral blood from $44 \%$ to $19 \%$ was seen as well as a reduction in leukocyte counts. Due to hematotoxicity and recurring infections the dose of sunitinib was reduced regularly and adjusted in the course of treatment. In December 2013 the AML progressed again and treatment with sunitinib was stopped. Cytoreductive therapy with hydroxyurea and after another rise of blast cells with cytarabine showed only shorttime response. The patient died from an infection in December 2013.

\section{Discussion}

The WHO classification and risk stratification according to European LeukemiaNet in AML is guided by genetic aberrations [10]. The presence of a FLT3-ITD is associated with a higher risk of relapse and inferior overall survival, therefore allogeneic transplantation is often used as postremission therapy. For elderly or unfit patients there are few options in relapsed FLT3-ITD positive AML. Clinical studies evaluating the use of FLT3 inhibitors are ongoing with midostaurin (PKC412), lestaurtinib, sorafenib, quizartinib (AC220), crenolanib and PLX3397 as single agents or in combination with chemotherapy [11].

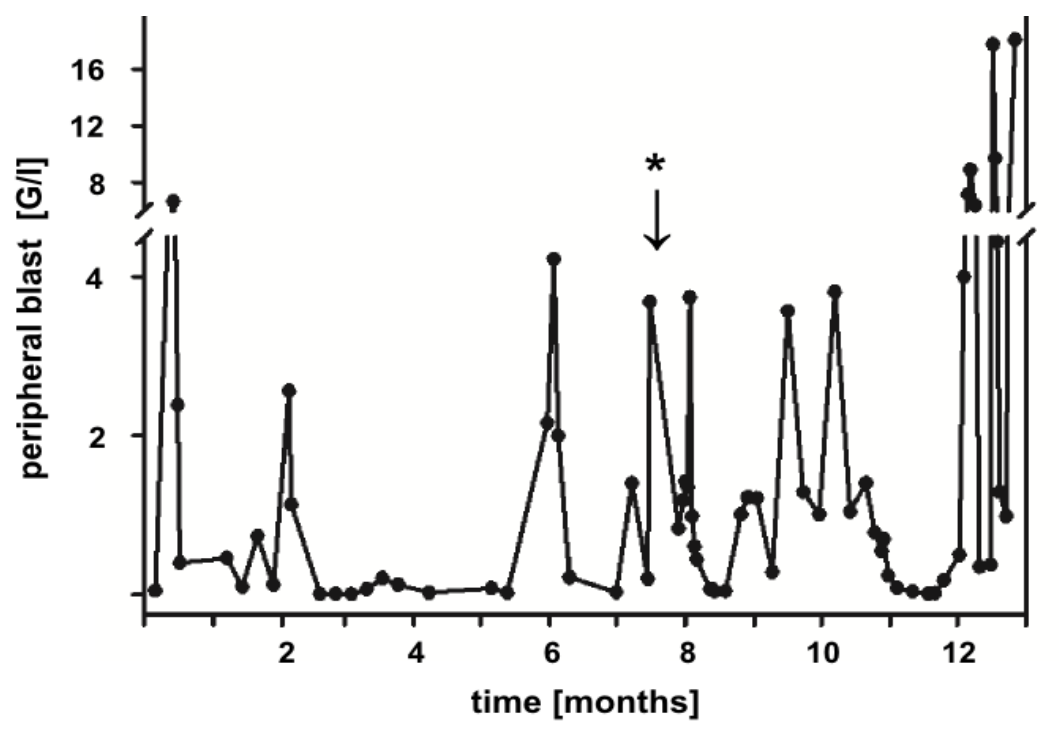

Sorafenib

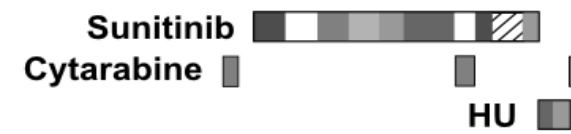

Figure 1: Development of peripheral blast count. 
A

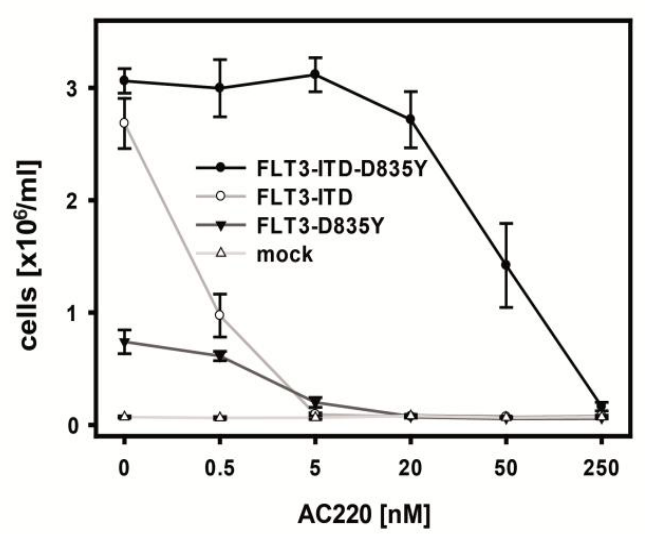

C

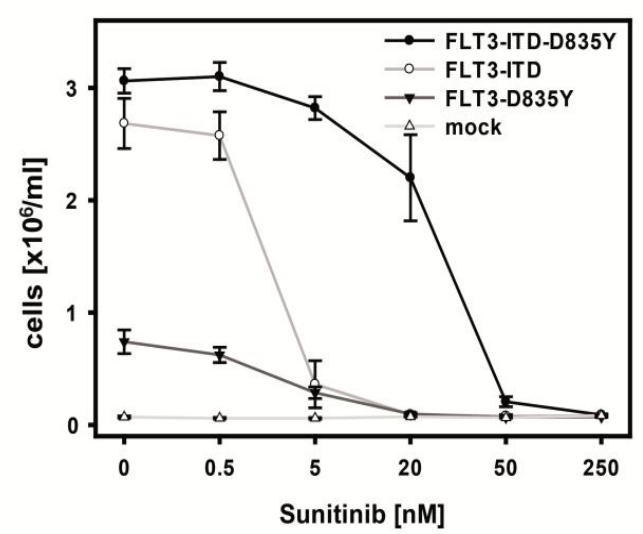

B

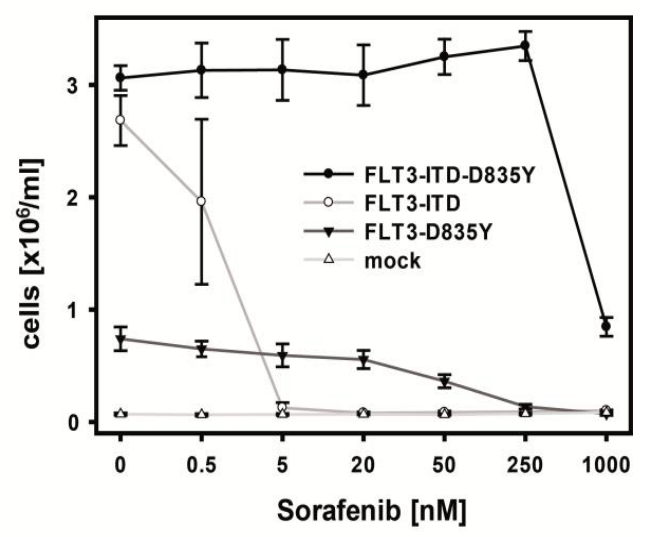

D

\begin{tabular}{|lccc|}
\hline IC $_{50}$ [nM] & Sorafenib & Sunitinib & AC220 \\
\hline FLT3-ITD-D835Y & $\mathbf{7 9 5}$ & $\mathbf{2 2}$ & $\mathbf{3 8}$ \\
\hline FLT3-ITD & 2.1 & 2.8 & $<0.5$ \\
\hline FLT3-D835Y & 49 & 3 & 2.3 \\
\hline
\end{tabular}

Figure 2: Treatment of FLT3 expressing cells with tyrosine kinase inhibitors.

The multi-kinase inhibitor sorafenib has shown promising results and is evaluated in clinical phase III trials, however resistance due to emergence of FLT3-D835 mutation can occur [5,12,13]. Sunitinib (SU11248) is a small-molecule RTK-inhibitor which is approved for the treatment of gastrointestinal stromal tumors and renal cell cancer. Moreover clinical activity in AML was seen especially in FLT3 mutated patients and after acquired resistance to sorafenib therapy $[4,9]$.

Our case report of an AML patient with concurrent FLT3-ITD and FLT3-TKD demonstrates that tyrosine kinase inhibitors are an option for a personalized therapeutic approach in selected patients that might prolong survival.

\section{References}

1. Thiede C, Steudel C, Mohr B, Schaich M, Schäkel U, et al. (2002) Analysis of FLT3-activating mutations in 979 patients with acute myelogenous leukemia: association with $F A B$ subtypes and identification of subgroups with poor prognosis. Blood 99: 4326-4335.

2. Janke H, Pastore F, Schumacher D, Herold T, Hopfner KP, et al. (2014) Activating FLT3 mutants show distinct gain-of-function phenotypes in vitro and a characteristic signaling pathway profile associated with prognosis in acute myeloid leukemia. PLoS One 9: e89560.

3. Opatz S, Polzer H, Herold T, Konstandin NP, Ksienzyk B, et al. (2013) Exome sequencing identifies recurring FLT3 N676K mutations in core-binding factor leukemia. Blood 122: 1761-1769.

4. Baker SD, Zimmermann El, Wang YD, Orwick S, Zatechka DS, et al. (2013) Emergence of polyclonal FLT3 tyrosine kinase domain mutations during sequential therapy with sorafenib and sunitinib in FLT3-ITD-positive acute myeloid leukemia. Clin Cancer Res 19(20): p. 5758-68.

5. Man CH, Fung TK, Ho C, Han HH, Chow HC, et al. (2012) Sorafenib treatment of FLT3-ITD(+) acute myeloid leukemia: favorable initial outcome and mechanisms of subsequent nonresponsiveness associated with the emergence of a D835 mutation. Blood 119(22): p. 5133-43.

6. Polzer H, Janke H, Schmid D, Hiddemann W, Spiekermann K (2013) Casitas B-lineage lymphoma mutants activate AKT to induce transformation in cooperation with class III receptor tyrosine kinases. Exp Hematol 41: 271-280.

7. Yates J, Glidewell O, Wiernik P, Cooper MR, Steinberg D, et al. (1982) Cytosine arabinoside with daunorubicin or adriamycin for therapy of acute myelocytic leukemia: a CALGB study. Blood 60: 454-462.

8. Büchner T, Hiddemann W, Berdel WE, Wörmann B, Schoch C, et al. (2003) 6 -Thioguanine, cytarabine, and daunorubicin (TAD) and high-dose cytarabine and mitoxantrone (HAM) for induction, TAD for consolidation, and either prolonged maintenance by reduced monthly TAD or TAD-HAM-TAD and one course of intensive consolidation by sequential HAM in adult patients at all ages with de novo acute myeloid leukemia (AML): a randomized trial of the German AML Cooperative Group. J Clin Oncol 21: 4496-4504.

9. Fiedler W, Serve H, Döhner H, Schwittay M, Ottmann OG et al. (2005) A phase 1 study of SU11248 in the treatment of patients with refractory or resistant acute myeloid leukemia (AML) or not amenable to conventional therapy for the disease. Blood 105: 986-993.

10. Döhner H, Estey EH, Amadori S, Appelbaum FR, Büchner $\mathrm{T}$, et al. (2010) Diagnosis and management of acute myeloid leukemia in adults: recommendations from an international expert panel, on behalf of the European LeukemiaNet. Blood 115: 453-474.

11. Wander SA, Levis MJ, Fathi AT (2014) The evolving role of FLT3 inhibitors in acute myeloid leukemia: quizartinib and beyond. Ther Adv Hematol 5: 65-77. 
Citation: Polzer H, Janke H, Schneider S, Hiddemann W, Subklewe M, et al. (2015) Individualized Treatment Strategy with Small-Molecular Inhibitors in Acute Myeloid Leukemia with Concurrent FLT3-ITD and FLT3-TKD Mutation. J Clin Case Rep 5: 622. doi:10.4172/2165-7920.1000622

12. Borthakur G, Kantarjian H, Ravandi F, Zhang W, Konopleva M, et al. (2011) Phase I study of sorafenib in patients with refractory or relapsed acute leukemias. Haematologica 96: 62-68.

13. Metzelder S, Wang Y, Wollmer E, Wanzel M, Teichler S, et al. (2009)
Compassionate use of sorafenib in FLT3-ITD-positive acute myeloid leukemia: sustained regression before and after allogeneic stem cell transplantation. Blood 113(26): p. 6567-71. 